\title{
Do We have Arguments in Decision Making: Conservative or Surgical Treatment in Painful Degenerative Large Rotator Cuff Tears?
}

Karl Vincent ( $\square$ vincentkarl347@gmail.com )

Universite Paris-Sud https://orcid.org/0000-0001-8850-389X

\section{Olivier Gagey}

Universite Paris-Sud

Charlotte Leboeuf-Yde

Universite Paris-Sud

\section{Research article}

Keywords: research article, rotator cuff, degeneration, surgical treatment, conservative treatment

Posted Date: November 23rd, 2020

DOI: https://doi.org/10.21203/rs.3.rs-111900/v1

License: (c) (i) This work is licensed under a Creative Commons Attribution 4.0 International License.

Read Full License 


\section{Abstract}

\section{Background and Hypotheses:}

The contemporary research literature indicates that there is no significant clinical difference in terms of improvement of pain and functional disability between surgical repair and rehabilitation treatment for degenerative rotator cuff tears. It could be hypothesized that rehabilitation, which is cheaper and causes few complications, is preferable for smaller lesions, whereas surgical repair might be needed for the larger lesions. The purpose of our review was to assess whether the size of the tear, particularly the large or massive, plays a major role in terms of long-term clinical results for rehabilitation compared to surgical repair.

\section{Method:}

We searched the literature on PubMed, Embase, PEDro, and Cochrane Library database up to March 2020 for randomized controlled clinical trials that compared long term results for surgical vs. rehabilitation concerning large or massive degenerative rotator cuff tears. A systematic, critical review process was planned using a slightly modified SIGN checklist. The review methodology followed the Preferred Reporting Items for Systematic Review and Meta-Analyses (PRISMA) guidelines and was submitted to PROSPERO and registered under the number CRD42020146161.

\section{Results:}

We found no randomized clinical trials that had dealt with this subject.

\section{Conclusion:}

There is no evidence that would help to choose between surgical repair or rehabilitation in case of large or massive rotator cuff degenerative tears. The reason for this is probably that large tears may be relatively painless since they often occur after retirement and therefore are relatively rarely seen in clinical practice. To obtain enough study subjects, it would therefore be necessary to study this question in multicenter randomized clinical trials.

\section{Background}

Shoulder pain is a major public health problem; it has been reported to affect up to $2 / 3$ of the general population over a lifetime (1). It can affect the quality of life (2), resulting in treatments (3), sick-leave (4), job loss, and early retirement (5). It is therefore important to provide a correct diagnosis and to match it with the most efficient treatment (4).

There are many possible diagnoses for the painful shoulder e.g. subacromial impingement syndrome, bursitis, adhesive capsulitis, biceps tendinopathy or tear, labral tear, glenohumeral or acromioclavicular joint arthritis, joint instability, and shoulder instability. However, the most commonly supposed cause of 
shoulder pain is degeneration of the rotator cuff tendons, which can progress to result in partial or complete tears (6). The rotator cuff consists of a group of muscles with their tendons that stabilize the shoulder. Of the seven scapulohumeral muscles, four make up the rotator cuff. These four muscles are the supraspinatus, the infraspinatus, teres minor, and the subscapularis muscle. This type of degeneration starts as, usually, silent 'tendinopathies', which may progressively worsen by tears of one or several of the tendons (3). These tears are classified into four types depending on their size, namely: small, medium, large, and massive tears (7). These defects are defined on the basis of the size of the antero-posterior tears (small: $<1 \mathrm{~cm}$ of the supraspinatus tendon; medium: $1-3 \mathrm{~cm}$ of the supraspinatus tendon; large: $3-5 \mathrm{~cm}$ including both the supra- and infraspinatus tendons, and massive: $>5 \mathrm{~cm}$ involving all three tendons).

Studies have shown that the natural history of this degenerative pathology has a strong positive correlation with age (8). Although the prevalence between degeneration and reported pain is parallel over time, the two trajectories separate at about the age of 65 , when the prevalence of pain diminishes but the prevalence of degeneration continues to increase (9). It is therefore possible that it is not definitely the degenerated or torn tendon that causes the pain.

This uncertainty regarding the cause of the pain may explain the two fundamentally different treatment approaches for this condition: conservative and surgical. Thus, the conservative treatment, mainly consists of rehabilitation with strengthening of the shoulder muscles that can be completed by antalgic and/or anti-inflammatory drugs and/or corticoid injections (10), whereas the surgical treatment aims at closing the tear by arthroscopy or open rotator cuff repair with or without debridement (11). Assuming that both are equally efficient, the first has the advantage of being cheaper and causing fewer complications than the second (12).

For clinicians and patients, it probably seems logical that rehabilitation should be used for the simpler cases and surgical repair saved for the more complicated, the more painful and the more degenerated tendons, those with the largest tears. However, it could also be argued that surgical repair at a relatively early stage could prevent an aggravation of the tear thus decreasing the need for further surgery perhaps at older age with the associated complications that this could entail.

However, the current literature does not seem to indicate clearly any significant clinical difference in terms of improvement of pain and functional disability between the two types of care (13). A recent systematic review showed that outcomes were similar for both treatment approaches (14). However, all these reviewed studies, except two randomized controlled clinical trials, were cohort studies without external control groups, and the studies consisting of conservatively treated patients generally included patients with small or medium sized tears, whereas the surgically treated patients typically presented with large or massive tears. It is therefore possible that the surgical group would have better results had the cases not been so severe and, conversely, that the conservative treatment would have had worse outcomes, if the case mix had included more severe tears. Further, the definition of tear size seems uncertain and authors 
did not report this item clearly in the review. It was also impossible to judge the reproducibility of this variable, as authors did not report quality control of their studies.

One of the outcome variables in this review (14) was 'need for future surgery'. However, it is possible that these two types of patients (those choosing rehabilitation treatment as compared to those choosing surgical repair) would react differently to the possibility of further surgery. Perhaps those who received only rehabilitation may have considered this treatment approach as a first attempt, assuming that if it would not work, there would be surgery to help afterwards. On the other hand, patients who were operated would perhaps consider the surgical option exhausted, if improvement was not sufficient for their liking, hence not returning for further surgery. This outcome variable would therefore not necessarily be a suitable measurement of treatment success.

Therefore, it is obvious that to compare the effectiveness of the two treatments, it would have been necessary to compare two intervention groups that are similar on the size of the tear using suitable outcome measurements.

For these reasons, we decided to make a new systematic and critical review of the literature, including only randomized controlled clinical trials that clearly described the study subjects in relation to the size of the tear both at baseline and in relation to the treatment outcome including any complications they may arise. The purpose of our review was to assess whether the size of the tear, specifically the large or massive, plays a major role in terms of the long-term clinical results for rehabilitation vs. surgical repair. Therefore, our study objective was to investigate if usual surgical treatment had better outcome than usual rehabilitation (exercise) treatment in large or massive degenerative tears of the rotator cuff.

\section{Method}

\section{Design and registration}

We intended to perform a systematic and critical review of randomized controlled clinical trials, comparing long term results for surgical repair vs. rehabilitation for large or massive degenerative rotator cuff tears. The review was submitted to PROSPERO and registered under the number CRD42020146161.

\section{Information sources and search}

Searches were made in the PubMed, Embase, PEDro and Cochrane Library databases to identify articles until March 2020. Two types of keywords were used: MeSH terms and free text and their subheadings. This search was made with the help of a librarian of Paris-Saclay University with regard to the search algorithm. The terms included rotator cuff injuries/surgery, rotator cuff tear, surgery, surgical procedures/operative, general surgery, surgery, outcomes, conservative, conservative treatment, and randomized clinical trial. The search concerned only English language and human studies.

Inclusion and exclusion criteria are described in the Table 1. 
Table 1

Eligibility criteria

\section{Inclusion criteria}

- Randomized controlled clinical trials that compare surgical repair vs rehabilitation.

- Studies in hospitals, clinics, rehabilitation centers.

- Pathologies concerning degenerative rotator cuff tears with patients over 50 years old.

- Studies that include and report on large or massive rotator cuff tears diagnosed by RMI, arthro-

scanner, or ultrasound.

- Studies that describe at least one of the following outcomes of the treatments: strength, pain, or function.

- Articles that describe the evolution and complications of the treatment i.e. aggravation of the tears diagnosed by RMI, arthro-scanner, ultrasound.

- For surgical articles: arthroscopy or open rotator cuff repair with or without debridement.

- For conservative treatment: rehabilitation with exercise with or without injections and/or antiinflammatory, antalgic drugs.

\section{Exclusion criteria}

- Studies that do not describe the type of the tears.

- Studies that include patients who had previous surgical repair of the rotator cuff.

- Studies that include patients who suffer from other pathologies than degenerative rotator cuff tears

i.e. traumatic causes or young patients.

- Duration of follow up of $<1$ year.

- Sample size at baseline $<50$.

The first author did a preliminary selection on the basis of titles and abstracts. Whole texts were read, when there were doubts about the inclusion and exclusion criteria. The inclusion criteria in the preliminarily selected articles were then controlled independently by one of the two other authors on the basis of the full texts. The opinion of the third author could be requested in case of disagreement on the inclusion of an article. We manually screened the references of each included study to ensure that no studies were missed. The Preferred Reporting Items for Systematic Review and Meta-Analyses (PRISMA) 2009 was followed and Fig. 1 shows the entire selection process and the reasons for exclusions.

\section{Data extraction}

The main outcomes of interest were changes in pain (VAS or other validated scores) and validated functional scores from baseline to the last available outcome, whether they were reported as exact estimates or differently, e.g. as odds ratios or relative risks. An additional outcome concerned the complication rate in each group. 
We intended to extract three types of information: i) descriptive information of included articles, and ii) information on internal validity and overall assessment of the study (Table 2). A specific checklist was established to assess the studies according to the methodological requirements for randomized controlled clinical studies. This checklist was based on existing recommendations in the SIGN statement (15). 
Table 2

Adapted checklist from SIGN Methodology checklist for randomized clinical trials.

Section 1: Internal validity

1. The study addresses an appropriate and clearly focused question.

2. The assignment of subjects to treatment groups is randomised.

() Yes, () no,

$\{$ can't say

3. An adequate concealment method is used.

4. The design keeps subjects and investigators 'blind' about treatment allocation.

5. The treatment and control groups are similar at the start of the trial.

() Yes, () no,

\} can't say

6. The only difference between groups is the treatment under investigation.

7. All relevant outcomes are measured in a standard, valid and reliable way (arthrography, MRI, ultrasound).

$\left\{\begin{array}{l}\text { Yes, ( ) no, } \\ \text { can't say }\end{array}\right.$

() Yes, () no,

( can't say

$\left\{\begin{array}{l}\text { Yes, ( ) no, } \\ \text { can't say }\end{array}\right.$

8. Was the percentage of the individuals or clusters recruited into each treatment arm of the study who dropped out before the study completion inferior to $20 \%$ ?

9. All the subjects are analysed in the groups to which they were randomly allocated (often referred to as intention to treat analysis).

10. Where the study is carried out at more than one site, results are comparable for all sites.

$\left\{\begin{array}{l}\text { Yes, ( ) no, } \\ \text { can't say }\end{array}\right.$

$\left\{\begin{array}{l}\text { Yes, () no, } \\ \text { can't say }\end{array}\right.$

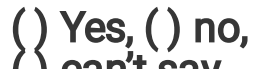

( can't say $\left\{\begin{array}{l}\text { Yes, ( ) no, } \\ \text { can't say }\end{array}\right.$

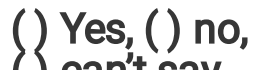
()an't say

() Does not apply

\section{Section 2: Overall assessment}

High quality

$(++) \leq$

2.1 How well was the study done to minimise bias?

2.2 Taking into account clinical considerations, your evaluation of the methodology

Acceptable

$(+) \leq$

used, and the statistical power of the study, are you certain that the overall effect is

due to the study intervention? (clinical considerations: Are the results clinically

different between the two groups? Is the complication rate superior in surgery or rehabilitation treatment?)

2.3 Are the results of this study directly applicable to the patient group targeted by this guideline?

2.4 Notes. Authors' conclusions. Add any comments on your own assessment of the study, and the extent to which it answers your question and mention any areas of uncertainty raised above. (authors' interpretation was consistent with results benefit and harm, time of follow-up)

Low quality

$(-) \leq$

Unacceptable

- reject $0 \leq$

( ) certain, () uncertain

( ) Yes, ( ) no

All outcome measurements and results (differences in the score, main effect, $95 \% \mathrm{Cl}$ or $\mathrm{p}$-values, when $95 \% \mathrm{Cl}$ were not reported or could not be computed), would be recorded directly into evidence tables. In 
addition, losses and exclusions would be noted and how the authors dealt with this.

Relevant information regarding study descriptions and method would be sought in the Methods section in the main text and information on results obtained from the Result section in the main text. Two readers would collect this information (KV and CLY), independently of each other, and in case of disagreement, discussions could be held with the third author (OG).

1 The list of descriptive items of included articles consisted of the following items:

- First author.

- Year of publication.

- Setting and location(s) where the study was performed.

- Sample size.

- Type of population at baseline.

- Interventions and types of treatment in the comparative groups.

- Time of follow up(s).

2 The quality assessment (SIGN Statement) included some minor changes. Concerning item 1.7 of Sect. 1 (Valid diagnostic tools for rotator cuff tears) we specified: RMI, arthro-scanner or ultrasound.

Item1.8 was changed from "What percentage of the individuals or clusters recruited into each treatment arm of the study dropped out before the study was completed? Yes/No/Can't say" to "Was the percentage of the individuals or clusters recruited into each treatment arm of the study who dropped out before the study completion inferior to $20 \%$ ? Yes/no/Can't say". This made it easier to answer the question and because, conventionally, a $20 \%$ drop out rate is regarded as acceptable (16).

\section{Data analysis and interpretation of findings}

Based on the above information, we aimed to distinguish the studies for which the overall quality was the best from those with lower quality, without introducing an exact cut-point. Nevertheless, we considered that if the response for the item 1.2 (The assignment of subjects to treatment groups is randomized) was "no" or "can't say", the study would be considered as unacceptable. Otherwise, the evaluation of studies would be based on an overall consideration of the credibility of data and obtained through consensus discussions, if necessary.

We would establish any difference in treatment outcome in each study and if this difference favored surgical repair or rehabilitation. We would then endeavor to calculate the odd ratios for this result and if possible, the number needed to treat. We would also calculate the number of reported complications in each study.

Concerning clinical significance, we planned to record if there were any differences between the groups, and if so, if these were statistically and clinically significant. 


\section{Results}

As can be seen in Fig. 1, we identified 178 studies (PubMed 95, Embase 33, Cochrane library 34 and PEDro 16). From these 178 studies we excluded 39 doubles. Of the remaining 139 articles, 134 were excluded on the basis of their title or abstract because they clearly did not fulfill the inclusion criteria. Of the remaining 4 randomized controlled clinical trials dealing with the topic (17)(18)(19)(20), none was found to be useful, because they did not report on large or massive rotator cuff tears.

\section{Discussion}

\section{Summary of findings}

Our literature review aimed to find randomized controlled clinical trials that compared surgical repair vs. rehabilitation for degenerative rotator cuff disorders and those dealing specifically with large and massive tears, to make it possible to investigate if this indication is more suitable for surgery than for conservative treatment. However, we found no studies that had focused their intervention on these specific lesions. Therefore, it is not possible to determine the best procedure to treat these lesions.

\section{Methodological considerations}

In relation to the completeness of our literature search, this cannot be fully guaranteed, as it is possible that some relevant articles had been left out. Nevertheless, we tried to minimize this by using MeSH terms and free terms and we were assisted by a librarian of Paris-Saclay University. We choose to identify articles with the PubMed database in addition to the Embase, PEDro and Cochrane Library databases, which has shown to give excellent results (21). It is also possible that there would have been some articles on this topic in another language than English.

\section{Discussion of findings}

The present status relating to the therapeutic efficacy of surgical repair vs. rehabilitation is that there is no difference between the two (11). However, our initial readings showed that information is based only on studies dealing with small or medium tears (14). The lack of clinical trials relating to large or massive degenerative rotator cuff tears is likely due to the fact that their frequency in clinic is lower than small or medium tears. The reason for this might be that they tend to occur later in life during a period of less activity, and therefore probably causing less pain e. Nevertheless, their management remains of major importance given the cost and risk for complications that are associated with the surgical approach.

It is therefore necessary to set up multicenter randomized clinical trials, which will make it possible to study a sufficient number of cases. Alternatively, random allocation could be stratified based on size, and results reported independently for each group in studies including all types of tears.

Clinicians probably consider the size of the lesion to be of importance in the choice of therapy. It is, therefore, unfortunate that the lack of specific studies on subgroups makes it impossible to decide which 
types of lesions should be treated how. Therefore, presently, the treatment approach has to be based on clinical experience and willful thinking.

\section{Conclusion}

There is, to our knowledge, presently no evidence on the type of treatment (rehabilitation or surgical repair) that is the best option for large or massive rotator cuff degenerative tears. Considering that such cases are rare, it would be necessary to conduct a well-designed multicenter randomized controlled clinical trial to compare treatment outcomes in terms of pain and function, cost, and long-term complications for this type of lesion.

\section{Abbreviations}

None

\section{Declarations}

Ethical approval and consent to participate:

Not applicable

Consent to participate:

Not applicable

Availability of data and materials:

Not applicable

Competing interests:

The Authors declare that there is no conflict of interest

\section{Funding:}

This study was funded by the Institut Franco-Européen de Chiropraxie, France. The funding source had no involvement in study design, analysis, interpretation, or manuscript preparation.

\section{Authors' contributions:}

$\mathrm{KV}$ formulated and reviewed search terms and searches with the help of a librarian Catherine Weil at Orsay University. KV distributed and organized articles for reviewers, screened and appraised articles, was the primary author of the manuscript, evidence tables and risk of bias table. CLY screened and appraised 
articles, reviewed and edited the manuscript and evidence Tables. OG reviewed and edited the manuscript and advised throughout the review process. All authors read and approved the final manuscript.

\section{Acknowledgements:}

We would like to thank university librarian Catherine Weil, Université Paris Saclay, for thorough assistance in searching the library databases.

\section{References}

1. Luime JJ, Koes BW, Hendriksen IJM, Burdorf A, Verhagen AP, Miedema HS, et al. Prevalence and incidence of shoulder pain in the general population; a systematic review. Scand J Rheumatol. 2004;33(2):73-81.

2. Minns Lowe CJ, Moser J, Barker K. Living with a symptomatic rotator cuff tear 'bad days, bad nights': a qualitative study. BMC Musculoskelet Disord. Jul 2014;15:228.

3. Linaker C, Walker-Bone K. SHOULDER DISORDERS AND OCCUPATION. Best Pract Res Clin Rheumatol. Jun 2015;29(3):405-23.

4. Marks $D$, Comans $T$, Bisset $L$, Thomas $M$, Scuffham PA. Shoulder pain cost-of-illness in patients referred for public orthopaedic care in Australia. Aust Health Rev. 15 oct 2018;

5. Wilson d'Almeida K, Godard C, Leclerc A, Lahon G. Sickness absence for upper limb disorders in a French company. Occup Med (Lond). Oct 2008;58(7):506-8.

6. Meislin RJ, Sperling JW, Stitik TP. Persistent shoulder pain: epidemiology, pathophysiology, and diagnosis. Am J Orthop. dec 2005;34(12 Suppl):5-9.

7. Lädermann A, Burkhart SS, Hoffmeyer P, Neyton L, Collin P, Yates E, and al. Classification of fullthickness rotator cuff lesions: a review. EFORT Open Rev. Dec 2016;1(12):420-30.

8. Moosmayer S, Gärtner AV, Tariq R. The natural course of nonoperatively treated rotator cuff tears: an 8.8-year follow-up of tear anatomy and clinical outcome in 49 patients. J Shoulder Elbow Surg. Apr 2017;26(4):627-34.

9. Vincent K, Leboeuf-Yde C, Gagey O. Are degenerative rotator cuff disorders a cause of shoulder pain? Comparison of prevalence of degenerative rotator cuff disease to prevalence of nontraumatic shoulder pain through three systematic and critical reviews. J Shoulder Elbow Surg. May 2017;26(5):766-73.

10. M J, S H, G L. EXERCISE THERAPY IN THE NON-OPERATIVE TREATMENT OF FULL-THICKNESS ROTATOR CUFF TEARS: A SYSTEMATIC REVIEW [Internet]. Vol. 13, International journal of sports physical therapy. Int J Sports Phys Ther; 2018 [cité 21 sept 2020]. Available on: https://pubmed.ncbi.nlm.nih.gov/30038823/

11. A Z, M D, G M, A O. A Comparison of the Clinical Outcomes between Arthroscopic and Open Rotator Cuff Repair in Patients with Rotator Cuff Tear: A Nonrandomized Clinical Trial [Internet]. Vol. 9, 
Advanced biomedical research. Adv Biomed Res; 2020 [cité 21 sept 2020]. Available on: https://pubmed.ncbi.nlm.nih.gov/32775306/

12. Piper CC, Hughes AJ, Ma Y, Wang H, Neviaser AS. Operative versus nonoperative treatment for the management of full-thickness rotator cuff tears: a systematic review and meta-analysis. J Shoulder Elbow Surg. March 2018;27(3):572-6.

13. Ryösä A, Laimi K, Äärimaa V, Lehtimäki K, Kukkonen J, Saltychev M. Surgery or conservative treatment for rotator cuff tear: a meta-analysis. Disabil Rehabil. 2017;39(14):1357-63.

14. Chalmers PN, Ross H, Granger E, Presson AP, Zhang C, Tashjian RZ. The Effect of Rotator Cuff Repair on Natural History. JB JS Open Access [Internet]. 9 feb 2018 [cité 1 août 2019];3(1). Available on: https://www.ncbi.nlm.nih.gov/pmc/articles/PMC6132904/

15. Harbour R, Miller J. A new system for grading recommendations in evidence based guidelines. BMJ. Aug 2001;323(7308):334-6.

16. Bell ML, Kenward MG, Fairclough DL, Horton NJ. Differential dropout and bias in randomised controlled trials: when it matters and when it may not. BMJ [Internet]. Jan 2013 [cité 19 oct 2019];346. Available on: https://www.ncbi.nlm.nih.gov/pmc/articles/PMC4688419/

17. Kukkonen J, Joukainen A, Lehtinen J, Mattila KT, Tuominen EKJ, Kauko T, and al. Treatment of nontraumatic rotator cuff tears: A randomised controlled trial with one-year clinical results. Bone Joint J. Jan 2014;96-B(1):75-81.

18. Kukkonen J, Kauko T, Virolainen P, Äärimaa V. The effect of tear size on the treatment outcome of operatively treated rotator cuff tears. Knee Surg Sports Traumatol Arthrosc. Feb 2015;23(2):567-72.

19. Lambers Heerspink FO, van Raay JJAM, Koorevaar RCT, van Eerden PJM, Westerbeek RE, van 't Riet $E$, and al. Comparing surgical repair with conservative treatment for degenerative rotator cuff tears: a randomized controlled trial. J Shoulder Elbow Surg. Aug 2015;24(8):1274-81.

20. Moosmayer S, Lund G, Seljom US, Haldorsen B, Svege IC, Hennig T, and al. Tendon repair compared with physiotherapy in the treatment of rotator cuff tears: a randomized controlled study in 103 cases with a five-year follow-up. J Bone Joint Surg Am. Sept 2014;96(18):1504-14.

21. Samadzadeh GR, Rigi T, Ganjali AR. Comparison of Four Search Engines and their efficacy With Emphasis on Literature Research in Addiction (Prevention and Treatment). Int J High Risk Behav Addict. 2013;1(4):166-71.

\section{Figures}




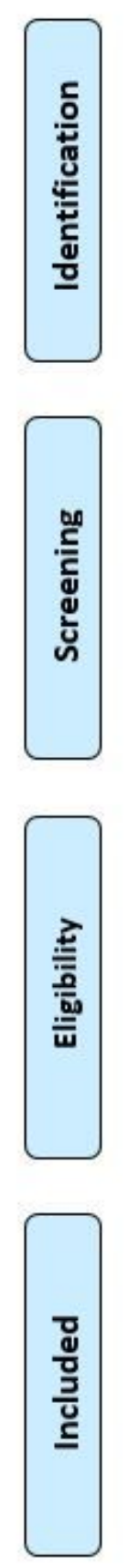
Records identified through database searching (PubMed=95, Embase=33, Cochrane $=34$, PEDro $=16$ ) $(n=178)$

Additional records identified through other sources

$$
(n=0)
$$

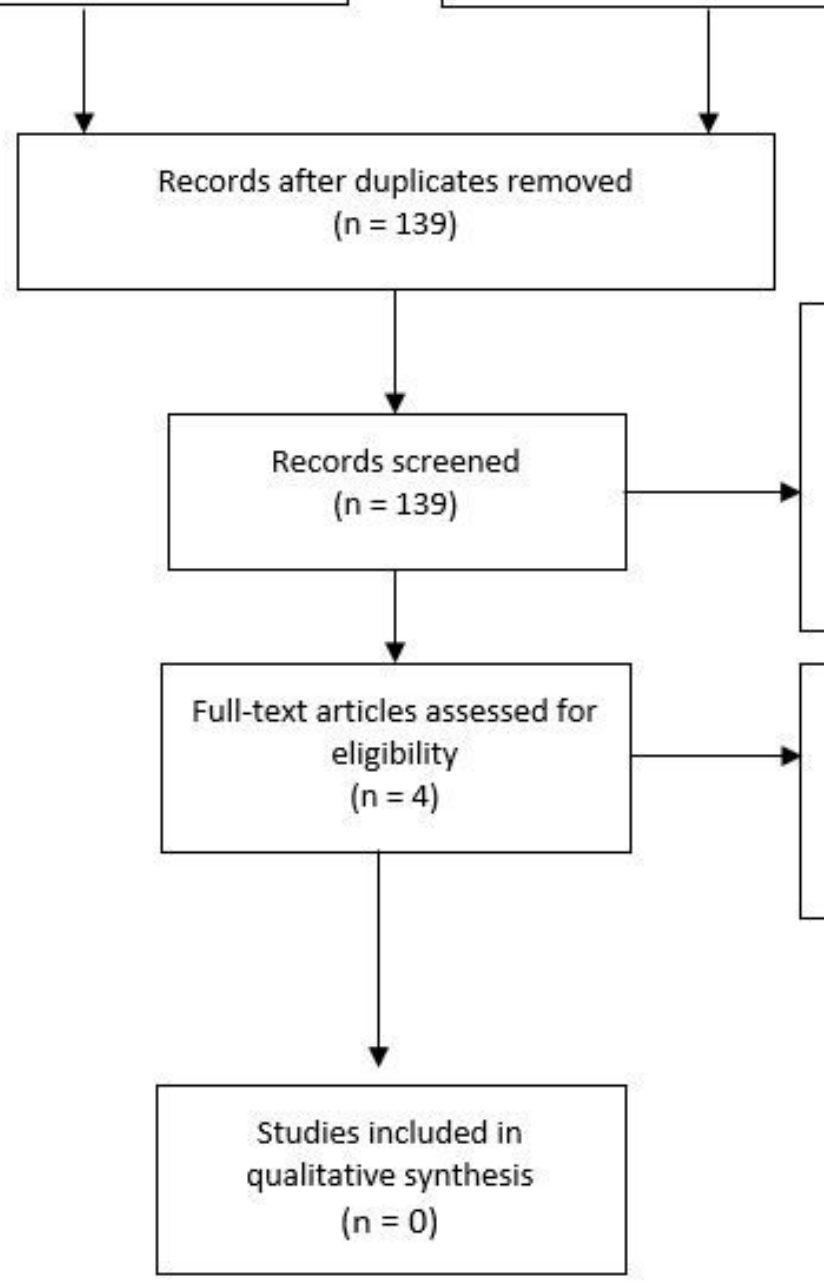

Records excluded on the basis of their title, abstract (RCTs that did not report on conservative versus surgery treatments, other designs) ( $n=135$ )

Full-text articles excluded, with reasons (none reported on large or massive tears)

$$
\text { ( } n=4)
$$

\section{Figure 1}

PRISMA diagram showing the result of application of the study algorithm to the number of studies included, with the number of studies removed with application of each exclusion criterion displayed. 


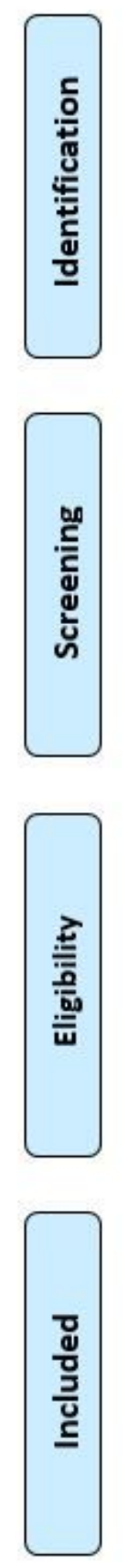
Records identified through database searching (PubMed=95, Embase $=33$, Cochrane $=34$, PEDro $=16$ ) $(n=178)$

Additional records identified through other sources

$$
(n=0)
$$
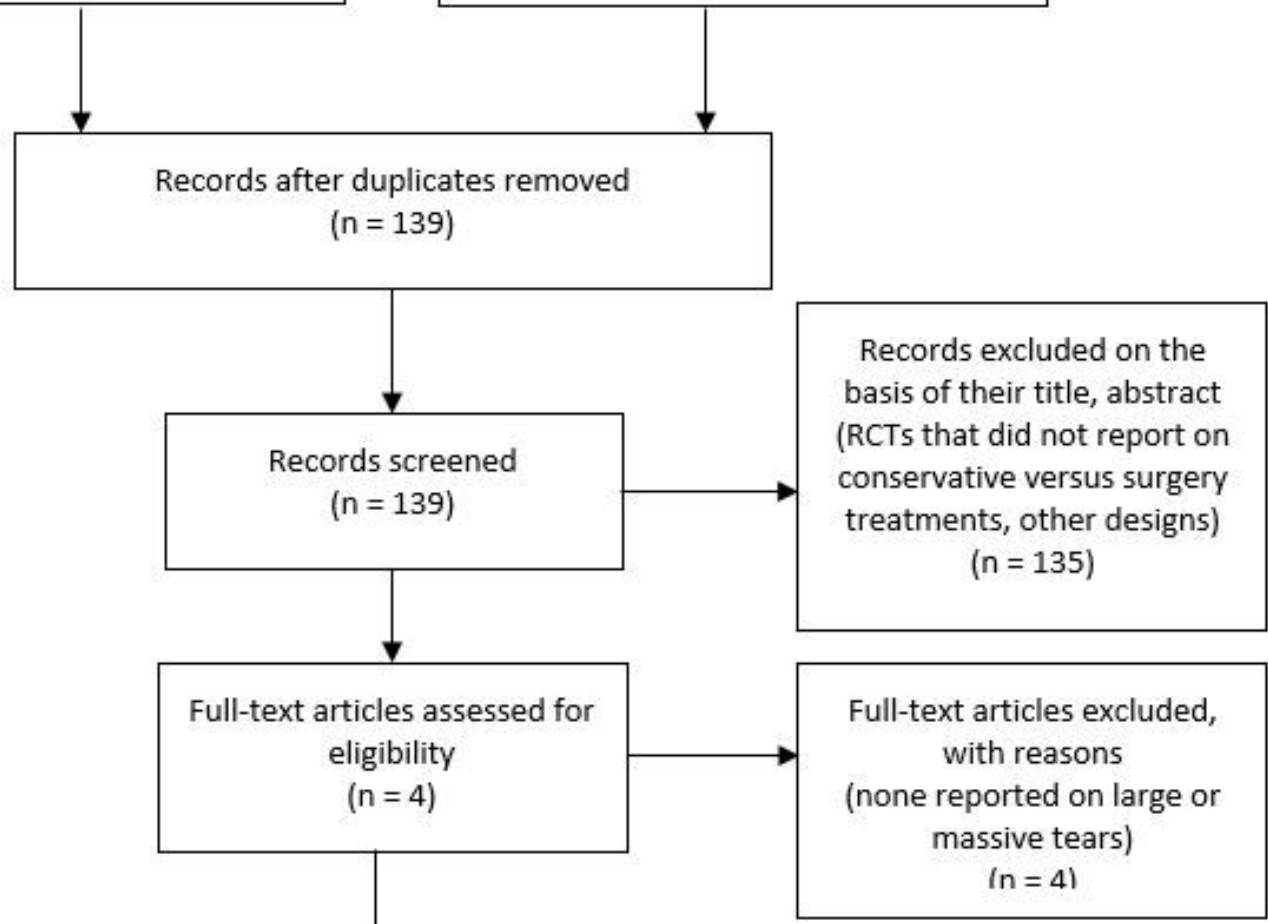

\section{Figure 1}

PRISMA diagram showing the result of application of the study algorithm to the number of studies included, with the number of studies removed with application of each exclusion criterion displayed. 